\title{
Home oxygen therapy during natural disasters: lessons from the great East Japan earthquake
}

\section{To the Editors:}

Oxygen therapy is given to patients with hypoxaemia caused by diseases such as chronic obstructive pulmonary disease, interstitial lung disease and cystic fibrosis [1]. In cases of natural disasters, patients receiving oxygen therapy in their homes can lose their stable oxygen supply for long periods of time due to power failure or equipment damage. Little has been established for the management of these patients during disasters. On March 11, 2011, a 9.0-magnitude earthquake and subsequent tsunami struck large parts of the east coast of Japan. Over 20,000 people died or were listed as missing following this disaster [2]. Here, we discuss the problems, based on our experience, in the management of patients receiving home oxygen therapy during disasters.

Avoiding treatment interruption during a disaster is essential in maintaining disease stabilisation for patients receiving oxygen therapy. In Japan, home oxygen therapy is widely used for patients with chronic respiratory failure and covered by the national healthcare insurance system [3]. Patients undergoing oxygen therapy are recommended to arrange for spare oxygen cylinders in case of electricity blackouts, and healthcare professionals, who manage respiratory care, can make such provisions in these cases. Since the Great Hanshin Earthquake of 1995, medical personnel and home oxygen service providers have recognised the importance of the management of oxygendependent patients during a disaster and have established emergency operation measures. The wide-scale disaster of March 2011, however, exceeded the presumed scenario.

In Ishinomaki, a port town in northeast Japan, more than 4,000 disaster victims visited the Japanese Red Cross Ishinomaki Hospital, a regional disaster medical centre, within a week of the earthquake. 70 patients receiving home oxygen therapy sought refuge at the hospital from March 11-14. Many offices of family physicians in Ishinomaki were destroyed by the tsunami. The interruption of traffic, causing isolation into divided regions, and the destruction of buildings prevented home oxygen providers from obtaining immediate access to sufficient oxygen cylinders for use during emergencies. The oxygen-dependent patients were, therefore, forced to seek refuge in the hospital.

All oxygen-dependent patients were triaged using the START (simple triage and rapid treatment) protocol [4] at the entrance hall of the hospital and brought into the outpatient ward. These patients received continuous oxygen supply via the medical gas central piping system. In the outpatient ward, the medical gas central piping system, the stocks of oxygen flow meters and room availability were insufficient for the unexpectedly high number of patients. Therefore, we contacted home oxygen providers via satellite phone and asked them to provide oxygen concentrators. On March 14, a temporary shelter was established inside the hospital using oxygen concentrators, and oxygen-dependent patients were transferred to the evacuation centre to receive oxygen therapy (fig. 1).
It is critical to prevent exacerbations in oxygen-dependent patients in order to avoid unnecessary critical care. Trained nurses were assigned to the evacuation centre to provide medical care and chest physicians took rounds in the area regularly. Appropriate infection prevention measures were applied to all patients and their families $[5,6]$. Patients were evaluated for their drug adherence because many of them lost their prescribed drugs during the tsunami. Patient medication notebooks, which are provided by the registered Japanese health insurance pharmacies [7], recorded all prescriptions and proved to be useful for identifying regular medications.

Despite these procedures, there was a high incidence of symptom exacerbation in oxygen-dependent patients. In total, 83 chronic pulmonary disease patients who were dependent on oxygen support, including eight patients who underwent combined noninvasive positive pressure ventilation with longterm oxygen therapy, visited the hospital during the 15-day follow-up period. Of these, $\sim 20 \%$ (17 out of 83 patients) experienced worsening of symptoms such as increased breathlessness, coughing, sputum production and high fever, thereby requiring additional treatments. None of these patients was found to have influenza. The most common causes of exacerbation were presumed to be tracheobronchial infection and treatment interruption. When the regional electrical power was restored, patients without symptom exacerbation returned home, and the evacuation centre for oxygen-dependent patients was closed on March 26. Patients who had been left homeless were transferred to nearby hospitals. Two patients in the terminal stage of chronic respiratory failure requiring ventilatory support at home underwent in-hospital care but died within 30 days of the disaster due to their exacerbations.

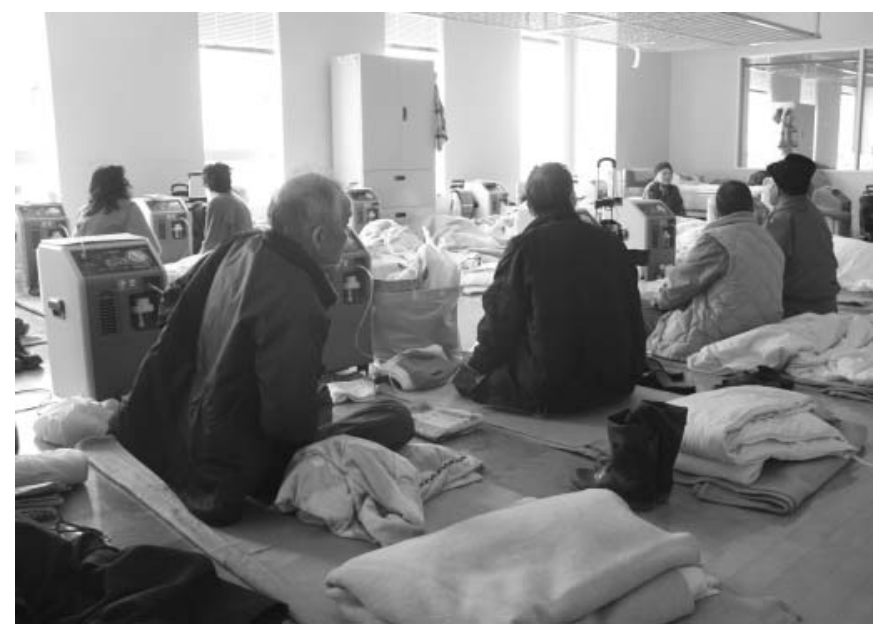

FIGURE 1. A temporary shelter for patients with oxygen therapy was established at the rehabilitation clinic of our hospital (Japanese Red Cross Ishinomaki Hospital, Ishinomaki, Japan). 
In summary, we emphasise that patients requiring home oxygen therapy need significant healthcare resources and should come to a hospital to seek medical care in the case of a natural disaster. Community-based plans for patients receiving home oxygen therapy are required. We conclude that emergency physicians, as well as experts from various fields, should develop strategies together for the management of patients receiving oxygen therapy during natural disasters.

\section{S. Kobayashi, M. Hanagama, S. Yamanda and M. Yanai}

Dept of Respiratory Medicine, Japanese Red Cross Ishinomaki Hospital, Ishinomaki, Japan.

Correspondence: S. Kobayashi, Dept of Respiratory Medicine, Japanese Red Cross Ishinomaki Hospital, 71 Nishimichishita, Hebita, Ishinomaki 986-8522, Japan. E-mail: skoba-thk@umin.ac.jp

Statement of Interest: None declared.

\section{REFERENCES}

1 Domiciliary oxygen therapy services: clinical guidelines and advice for prescribers. London, Royal College of Physicians, 1999.
2 National Police Agency of Japan. Damage situation and police countermeasures associated with 2011 Tohoku district - off the Pacific Ocean Earthquake. www.npa.go.jp/archive/keibi/biki/ higaijokyo_e.pdf Date last updated: August 24, 2011. Date last accessed: August 25, 2011.

3 Kida K. Home oxygen therapy in Japan: clinical application and considerations for practical implementation. JMAJ 2011; 54: 99-104.

4 Benson M, Koening KL, Schultz CH. Disaster triage: START, then SAVE - a new method of dynamic triage for victims of a catastrophic earthquake. Prehosp Disaster Med 1996; 11: 117-124.

5 Centers for Disease Control and Prevention. Infection Control Guidance for Community Evacuation Centers Following Disasters. www.bt.cdc.gov/disasters/commshelters.asp Date last updated: September 13, 2005. Date last accessed: August 25, 2011.

6 Rebman T, Wilson R, Alexander S, et al. Infection Prevention and Control for Shelters During Disasters. www.apic.org/ Content/NavigationMenu/EmergencyPreparedness/SurgeCapacity/ Shelters_Disasters.pdf Date last updated: 2007. Date last accessed: August 25, 2011.

7 Okumura J, Nishita Y, Kimura K. [Pharmaceutical supply for disaster victims who need chronic disease management in region with aging population based on lessons learned from the Noto Peninsula Earthquake in 2007]. Yakugaku Zasshi 2008; 128: 1275-1283. 